\title{
The Effect of Random Supply Shortages on the Economic Order Quantity Model
}

\author{
Fadi Asrawi ${ }^{1} \&$ Nazim Noueihed ${ }^{2}$ \\ ${ }^{1}$ Faculty of Business Administration and Economics, Haigazian University, Lebanon \\ 2 Department of Mathematics, Division of Mathematical Sciences, faculty of Arts \& Sciences, Haigazian \\ University, Lebanon \\ Correspondance: Fadi Asrawi, Professor, Faculty of Business Administration and Economics, Haigazian \\ University, Mexique Street, Kantari, Beirut, Lebanon. E-mail: fadi.asrawi@haigazian.edu.lb
}

Received: November 28, 2014

Accepted: December 19, 2014

Online Published: January 20, 2015

doi:10.5539/ijbm.v10n2p150

URL: http://dx.doi.org/10.5539/ijbm.v10n2p150

\begin{abstract}
In this paper, we will be considering a case of the subject of imperfection or deficiency in the inventory process of a single commodity which has many components. For example, the deterioration of items in the stock is one component; another component is the quality of the items received in an inventory order. This has been investigated by many like Yano and Lee (1995) and silver (1976), where the imperfection of the order was due to defectiveness of items and shortages on the supply side with the amount of imperfection as a discrete random variable. We shall consider only the case of shortages of supply, but allow for continuous random variables by taking the proportion of shortages relative to the order quantity. A mathematical model is developed where an analytic expression for the economic order quantity is obtained. Special cases for the distribution of the proportion will be presented, along with numerical examples and comparisons to the classical model without shortages.
\end{abstract}

Keywords: Inventory, order quantity, total cost, probability distribution, proportion of shortages, purchasing cost, set up cost, demand rate, supply shortage

\section{Introduction and Background}

The main assumption of the Economics Order Quantity (EOQ) model is that the order quantity is free of defects. However, the production environment has proven the inaccuracy of this assumption. As a result, many researchers have developed models that deal with imperfect quality or defective items.

The subject of imperfect quality of inventory orders has been investigated by many researchers. Silver (1976) considered both defective items and shortages on the supply side. Rosenblatt and Lee (1986) proposed a model that contains defective production after a random variable time point, Yano and Lee (1995) considered defective items in the order where it was assumed to be a random variable and review the work done on the random yield model. Cardenas-Barron (2000) corrected the optimal order quantity expression and Goyal and Cardenas-Barron (2002) proposed a near approximation for order quantity. Papachristos and Konstantaras (2006) studied the issue of non-shortages in model with proportional imperfect quality, when the proportion of the imperfects is a random variable. Salameh and Jaber (2000) developed a model of defective items where these are identified immediately at a cost that is added to the components of the total inventory cost. For them imperfect quality items can be sold as a single batch by the end of the screening procedure. A flow in their model was corrected by Maddah and Jaber (2007). They analyzed the effect of screening speed and variability of the supply process on the order quantity, and showed that the order quantity is larger than that of the classical EOQ model when the variability of the yield rate is practically low.

These models considered the imperfection in the ordered lot a discrete random variable with either a Poisson or Binomial distribution.

Jaggi and Mittal (2010) investigated the effect of deterioration on the retailer's economic order quantity when the items are of imperfect quality. They assumed the screening rate to be more than the demand rate. This enabled the retailer to fulfill the demand out of the perfect quality products along with the screening process. They found that in the case of highly deteriorating products the retailer should order more frequently to reduce his loss due to deterioration. When the defective items increase and profits decrease, the retailer needs to take corrective 
measures in order to get good quality products or change his source of supply.

Chang and Ho (2010) applied the renewal reward theorem to calculate the expected profit per unit time associated with Konstantaras et al model (2007). A simpler expression of the profit function was obtained using AGMI theorem to determine the optimal inventory policy. They showed that a larger total profit per unit time can be realized as the value of demand or the inspection rate increases. The cost of sending acceptable items to the working inventory income will decrease either the holding cost or the expected defective rate.

Gani and Maheswari (2010) proposed a fuzzy model of an inventory problem with imperfect items where backorders are allowed. The demand, holding cost, ordering cost, shortage cost and defective rate were taken as fuzzy triangular numbers. The Graded Mean Integration defuzzification method was used to find the total profit per unit time and derive the optimal order quantity to maximize total profit. They showed that the economic order quantity and backorder quantity and total profit increased when demand increases if all the costs and defective rate remain the same. They showed that economic production quantity, shortage quantity and total profit increase when the percentage of imperfect quality alone changes.

Wee, Wang and Yang (2012) investigated an EPQ model with imperfect quality items, shortage backordering and screening constraint. They showed that the rate to fulfil the backorders is dependent on the screening rate. If production and screening are synchronized, they demonstrated that the optimal production time is always greater than the optimal time to eliminate backorders.

In our model, we will consider that the supplier has a screening process of his own and sends good items. However, due to random reasons, there could be some shortages or less quantity than originally ordered. The situation we are considering is not far from reality for many reasons: increase in demand for the item, breakdown of the production machine in the factory, releases of new edition of the item where the production of the old one stops.

To allow for easier analysis, and more interesting cases about the distribution of shortages, we shall introduce a variable $\alpha=\frac{x}{q}$ where " $\mathrm{x}$ " is the amount of shortage and " $\mathrm{q}$ " the quantity ordered, and consider " $\alpha$ " to be a continuous random variable.

A mathematical model is developed and an analytic expression for the economic order quantity is obtained. Also special distributions along with numerical examples and comparisons will be presented.

\section{Research Methodology}

We shall adopt the usual traditional ways of investigating the specific inventory model we are considering. First we shall make reasonable physical and economic assumptions that are realistic and describe the model in a way so that the corresponding mathematical model mimics the real situation and has a feasible solution. The solution obtained shall be a unique optimal solution. After that, a variety of samples of the model are tested against classical models that have been studied before through a series of numerical examples that clearly show the effect of the factors of deficiency of the inventory process we considered.

\section{The Mathematical Model}

In order to reach a satisfactory mathematical model for the situation we have described, we shall make the following assumptions;

1) The replenishment of the inventory stock is instantaneous. This is not to add more complications to the model.

2) No shortages are allowed, also no backlogging.

3) The payment of the cost of the transaction or the quantity ordered is based on what is received and not what is ordered.

4) The demand rate per unit time or the depletion rate of the on hand inventory stock is constant.

As in most classical models used to represent the inventory stocks, a quantity "q" is ordered and depleted according to the constant demand rate, and the replenishment of the inventory stock is made in such a way to avoid the situation of shortages. We shall also assume that the selling price of the item during the depletion period is constant. This makes the optimization process use the total cost per unit per unit time function instead of the profit function. The formal function that we use as our objective function is the sum of three component functions: The set up cost or fixed cost, the holding cost, and the procurement cost which is the cost of the inventory quantity received.

In deterministic models, the objective function which is the total cost per unit per unit time is minimized to find 
the economic order quantity (EOQ). Some models yield an analytic expression for such a quantity, other models, with more complex conditions, demand numerical solutions and a theoretical proof of the existence and uniqueness of the optimizing order quantity.

Our model is not deterministic due to the randomness of the quantity supplied; the objective function is from a population of functions with a probability distribution induced by the distribution of the parameter $\alpha$. The question is what to optimize?

Many researchers, who tackled nondeterministic inventory models before, chose to minimize the expected objective function due to the fact that the joint distribution of the random parameters in the model induces a probability distribution on a class of objective functions. However Maddah and Nasr pointed out that according to Ross, this is a birth renewal process and what should be minimized is the expected total cost divided by the expected inventory period. We will adopt this procedure in our paper.

We shall also start by considering that the model is described by an initial value problem that is: a first order differential equation with the initial value representing the quantity ordered . We will then proceed to find an analytic expression for the objective function suggested by Ross that is : the expected total cost function divided by the expected inventory period. We will derive a formula for the economic order quantity that we call $q_{o}$. After that we shall consider five different distributions for the parameter $\alpha$, and consider several numerical examples by setting a specific distribution of the family. We will make a comparison of our model to the classical inventory model

* q: The quantity ordered;

* $\mathrm{x}$ : The random variable representing the amount of the shortage in the order;

* c: Purchasing cost / unit;

* h: Holding cost / unit / unit time;

* k: Set up cost / order;

*d: Demand rate / unit time.

An order of size " $\mathrm{q}$ " is placed. But due to random factors on the supply side $(\mathrm{q}-\mathrm{x})$ is received where $\mathrm{x}$ is a random variable. To simplify matters, let $\frac{x}{q}=\propto$, and consider $\alpha$ to be continuous random variable with a support on $[0,1]$. In real life cases, the support is $(0, \beta)$, where $\beta \leq 0.15$. It should be noted that the cost of the missing $\mathrm{X}$ units will not be held, and the payment is only for the $(\mathrm{q}-\mathrm{x})$ units received.

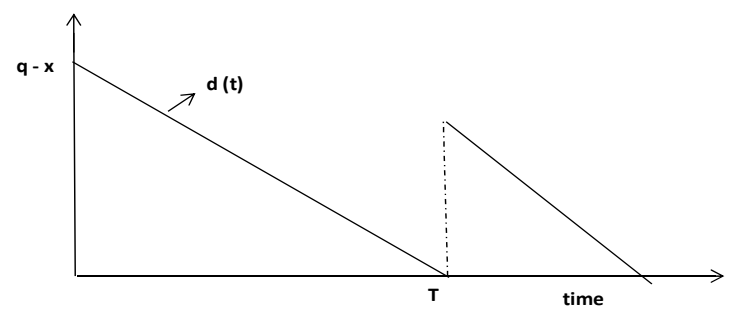

Let $\mathrm{d}(\mathrm{t})$ be the inventory level in time. $\mathrm{d}$ satisfies the (IVP):

$$
\frac{d d}{d t}=-D \text { and } d(o)=q-x=(1-\propto) q
$$

$d(t)=-D t+(1-\alpha) q$

The order cycle " $T$ " is a random variable obtained by setting $d(t)=0$

$T=(1-\alpha) q / D$

The holding cost is:

$$
\begin{aligned}
& H(q)=\left[\int_{0}^{\frac{\left(1-\alpha^{2}\right) q}{D}}[-D t+(1-\propto) q] d t\right] \\
& =h\left(-\frac{D t^{2}}{2}+\left.(1-\propto) q t\right|_{0} ^{\frac{(1-\alpha) q}{D}}\right.
\end{aligned}
$$




$$
=\frac{h(1-\propto)^{2} q^{2}}{2 D}
$$

The total cost is:

$$
C(q)=K+c(1-\propto) q+\frac{h(1-\propto)^{2} q^{2}}{2 D}
$$

According to the theorem of Ross, this is a renewal process, so to get optimal order quantity we should minimize the function:

$$
\begin{aligned}
g(q) & =\frac{E(C(q))}{E(T)} \\
& =\frac{K+C q E(1-\propto)+\frac{h q^{2}}{2 D} E\left((1-\propto)^{2}\right)}{\frac{q}{D} E(1-\propto)} \\
& =\frac{D k}{E(1-\alpha) q}+C D+\frac{h q E\left((1-\alpha)^{2}\right)}{2 E(1-\alpha)} \\
\rightarrow \quad \frac{d g}{d q} & =\frac{-D K}{E(1-\alpha) q^{2}}+\frac{h E\left((1-\propto)^{2}\right)}{2 E(1-\alpha)} \\
\rightarrow \quad & \frac{d g}{d q}=0 \rightarrow \frac{-D K}{q^{2}}+\frac{h E\left((1-\alpha)^{2}\right)}{2}=0 \\
\rightarrow \quad q^{2} & =\frac{D K}{h E\left((1-\propto)^{2}\right)} \\
\rightarrow \quad & q_{0}^{*}=\sqrt{\frac{2 D K}{h E\left((1-\alpha)^{2}\right)}}
\end{aligned}
$$

$q_{0}^{*}$ is the optimal order quantity for our model.

Note that if $\propto=0$, the formula reduces to $q_{c}^{*}=\sqrt{\frac{2 D K}{h}}$ being the order quantity without shortages.

Now $E\left((1-\propto)^{2}\right)=1-2 E(\propto)+E\left(\propto^{2}\right)$

$$
=1-2 \mu+\mu^{2}+\sigma^{2}
$$

With $\mu$ and $\sigma^{2}$ being the mean and variance of $\propto$.

Some special cares for the distribution of $\propto$.

\section{Proof of the Uniqueness of the Optimal Quantity}

We shall employ the convexity principle for the uniqueness of the minimizer of our objective function.

The second order derivative of the function is:

$\underline{\mathrm{d}^{2} \mathrm{~g}}=2 \underline{\mathrm{DK}}>0$, for all $\mathrm{q}>0$

$\mathrm{dq}^{2} \mathrm{q}^{3}$

This implies that the function "g" is convex, and since it is continuous, then the uniqueness of the optimizer follows.

Case 1: $\propto$ is uniformly distributed over $(a, b)$ with

$$
0 \leq a<b<1
$$

In this case, $E\left((1-\propto)^{2}\right)=1-\frac{2(a+b)}{2}+\frac{(a+b)^{2}}{2}+\frac{(b-a)^{2}}{12}=1-a-b+\frac{a^{2}+b^{2}+a b}{3}$ 


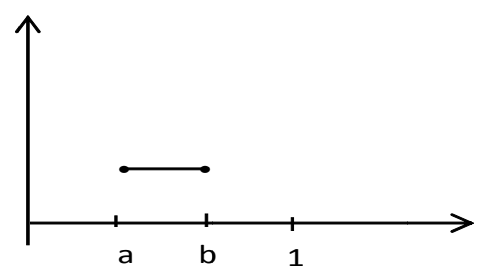

Numerical example1:

Let $\mathrm{h}=\$ 0.1 \mathrm{~K}=\$ 100 \quad \mathrm{~K}=\$ 100 \mathrm{D}=5$

And $(\mathrm{a}, \mathrm{h})=(0,0.05)$

$E\left((1-\propto)^{2}\right)=1-0.05+\frac{5(0.05)^{2}}{3}=0.95416$

The classical order quantity is:

$$
q_{c}^{*}=\sqrt{\frac{2(100) 5}{0.1}}=100 \text { units }
$$

\section{Our order quantity is:}

$$
q_{o}^{*}=\sqrt{\frac{2(100) 5}{0.95416}}=102.4 \text { units }
$$

Numerical example 2:

Let $\mathrm{h}=\$ 0.05 \quad \mathrm{k}=\$ 200 \quad \mathrm{D}=6$

The classical order quantity is:

$q_{c}^{*}=219$

\section{Our order quantity is:}

$q_{o}^{*}=224$

\section{Case 2:}

$\alpha$ has a triangular symmetric distribution with base (0. b), b $\angle 1$

The Probability Density Function (PDF) of $\alpha$ is:

$\frac{4 \propto}{b^{2}}, 0<\propto<\frac{b}{2}$

$P(\propto)=\left\{\frac{-4 \propto}{b^{2}}+\frac{4}{b}, \frac{b}{2} \leq \propto<b\right.$

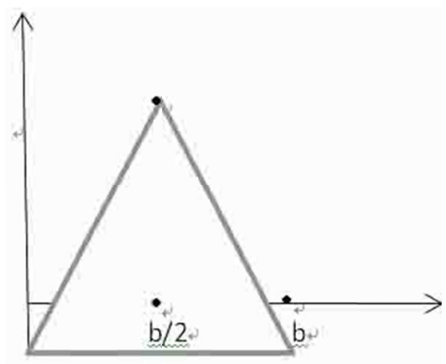




$$
E\left((1-\propto)^{2}\right)=1-b+\frac{7}{24} b^{2}
$$

Numerical example 3:

Let $\mathrm{h}=\$ 0.1 \mathrm{~K}=\$ 500 \mathrm{D}=5$ and $\mathrm{bb}=0.05$

$$
E\left((1-\propto)^{2}\right)=0.9507
$$

The classical order quantity is: $q_{c}^{*}=\sqrt{\frac{2(500) 5}{0.1}}=223.6$

Our model order quantity is: $q_{o}^{*}=\sqrt{\frac{2(500) 5}{0.1(0.9507}}=229.3$

Numerical example 4:

Let $\mathrm{h}=0.05 \quad \mathrm{~K}=500 \quad \mathrm{D}=5$

The classical order quantity is: $q_{c}^{*}=316$

Our model order quantity is: $q_{0}^{*}=324$

\section{Case 3}

$\alpha$ has a normal distribution with $\mu=0.05$ and $\sigma^{2}=0.01 E\left((1-\propto)^{2}\right)=0.9095$

Numerical example 5:

$\mathrm{h}=\$ 0.1 \mathrm{~K}=500 \mathrm{D}=10$

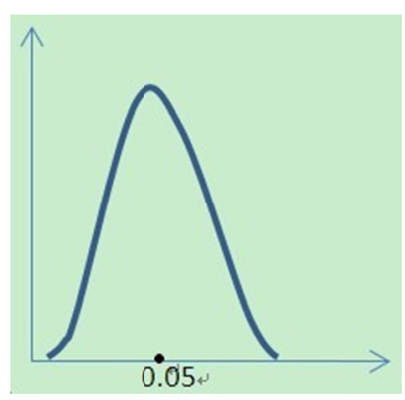

The classical order quantity is:

$q_{c}^{*}=316$;

Our model order quantity is: $q_{0}^{*}=331$;

Numerical example 6:

$\mathrm{h}=0.05 \quad \mathrm{~K}=500 \quad \mathrm{D}=10$

The classical order quantity is: $q_{c}^{*}=447$;

Our model order quantity is: $q_{0}^{*}=466$;

Case 4:

$\alpha$ has the $\mathrm{pdf}=\mathrm{f}(\alpha)=\{2 \alpha, 0<\alpha<10$, otherwise

$\mathrm{E}\left((1-\alpha)^{2}\right)=1-2 \mathrm{E}(\alpha)+\mathrm{E}\left(\alpha^{\prime}\right)$

$E(\alpha)=\int_{0}^{1} 2 \alpha^{2} d \alpha=\left.\frac{2 \alpha^{3}}{3}\right|_{0} ^{1}=\frac{2}{3}$

$E\left(\alpha^{2}\right)=\int_{0}^{1} 2 \alpha^{3} d \alpha=\left.\frac{\alpha^{4}}{2}\right|_{0} ^{1}=\frac{1}{2}$

$\Rightarrow E(1-\alpha)^{2}=1-\frac{4}{3}+\frac{1}{2}=\frac{1}{6}$

Numerical example $7: \mathrm{h}=\$ 0.1, \mathrm{k}=-\$ 500, \quad \mathrm{D}=5$

$\mathrm{q}_{\mathrm{c}}=223$ 
$\mathrm{q}^{*}=547$

Numerical example $8 \mathrm{~h}=\$ 0.1, \mathrm{k}=-400, \mathrm{D}=7$

$\mathrm{q}^{*} \mathrm{c}=236$

$\mathrm{q}^{*}=579$

Case 5: $\alpha$ has the slanted triangular distribution between 0 and $b$, in this case the pdf is $\mathrm{f}(\alpha)$

$\begin{aligned}= & \left\{\frac{-2 \alpha}{b^{2}}+2 b, 0 \prec \alpha \prec b\right. \\ & 0, \text { otherwise }\end{aligned}$

$E(\alpha)=\int_{0}^{b}\left(\frac{-2 \alpha^{2}}{b^{2}}+\frac{2 \alpha}{b}\right) d \alpha=\frac{-2 \alpha^{3}}{3 b^{2}}+\left.\frac{\alpha^{2}}{b}\right|_{0} ^{b}=\frac{-2 b^{3}}{3 b^{2}}+b=\frac{b}{3}$

$\mathrm{E}\left(\alpha^{2}\right)=\int_{0}^{b}\left(\frac{-2 \alpha^{3}}{b^{2}}+\frac{2 \alpha^{2}}{b}\right) d \alpha=\frac{-\alpha^{4}}{2 b^{2}}+\frac{2 \alpha^{3}}{3 b} \mid \|_{0}^{b}=-\frac{b^{2}}{2}+\frac{2 b^{2}}{3}=\frac{b^{2}}{6}$

$\left.\Rightarrow E(1-\alpha)^{2}\right)=1-\frac{2 b}{3}+\frac{b^{2}}{6}$

Let $\mathrm{h}=0.1$

$\Rightarrow E(1-\alpha)^{2}=1-\frac{(0.2)}{3}+\frac{(0.1)^{2}}{6}=0.953$

Numerical example 9: $\mathrm{h}=\$ 0.1, \mathrm{k}=-600, \mathrm{D}=8$

$\mathrm{q}_{\mathrm{c}}^{*}=310$

$\mathrm{q}^{*}=317$;

Numerical example 10: $\mathrm{h}=\$ 0.2, \mathrm{k}=-600, \mathrm{D}=10$

$\mathrm{q}^{*} \mathrm{c}=223$

$\mathrm{q}^{*}=229$;

It is obvious that there is a difference between the classical quantity ordered and the one due to shortages, and the difference becomes larger for larger quantities ordered or when the support of the probability distribution pdf the shortages proportion increases. Definitely this should be reflected as a saving in the overall cost of carrying the inventory of the item.

\section{Conclusion}

Mathematical models that treat imperfect quality of inventory orders considered that the imperfection of the order was due to defectiveness of items and shortages on the supply side with the amount of imperfection as a discrete random variable.

In our model we have considered five possible distributions for the parameter " $\alpha$ ". Most of them are realistic or close to the real market conditions. The numerical examples showed a difference between ordering under the situation of shortages on the supply ride and the classical inventory model. These differences are to become larger as the order quantity increases in size. This implies that for large quantity orders there is a substantial difference between the total cost/unit time according to the classical model and the total/unit time for the supply-shortages model. It is worthy to remark that if the shortage parameter " $\alpha$ " has some empirical distribution, constructed from actual market condition, the essential factor in our formula $\mathrm{E}\left((1-\alpha)^{2}\right)$ can be easily obtained from the histogram and substituted into the formula.

Our model considered the case of shortages of supply due to random reasons but allow for continuous random variables. Our mathematical model shows that the economic order quantity obtained is quite different than those in the classical order quantity with a better rate of accuracy. This reflects on the total cost for carrying inventory stocks for a long period of time. However we have to mention that in the case of the end of the conditions that promote the shortages, one should go back to the appropriate inventory model that well fits the situation. May be 
future models will consider further more realistic additions to the condition of supply shortages.

\section{References}

Cárdenas-Barrón, L. E. (2000). Observation on: Economic production quantity model for items with imperfect $\begin{array}{lllll}\text { quality. International Journal of Production Economics, } & 67(2) . & 201 .\end{array}$ http://dx.doi.org/10.1016/S0925-5273(00)00059-1

Goyal, S. K., \& Cárdenas-Barrón, L. E. (2002). Note on: Economic production quantity model for items with imperfect quality. A practical approach. International Journal of Production Economics, 77(1), 85-87. http://dx.doi.org/10.1016/S0925-5273(01)00203-1

Maddah, B., \& Jaber, M. Y. (2008). Economic order quantity for items with imperfect quality: revisited, International Journal of Production Economics, 112(2), 808-815. http://dx.doi.org/10.1016/j.ijpe.2007.07.003

Nagoor Gani, A., \& Maheswari, S. (2010). Advances in Fuzzy Mathematics.

Papachristos, S., \& Konstantaras, I. (2006). Economic ordering quantity models for items with imperfect quality. International Journal of Production Economics, 100, 148-154. http://dx.doi.org/10.1016/j.ijpe.2004.11.004

Rosenblatt, M., \& Lee, H. (1986). Economic production cycles with imperfect production processes. IIE Transactions, 18, 48-55. http://dx.doi.org/10.1080/07408178608975329

Ross, S. M. (1996). Stochastic Processes (2nd ed.). New York, N.Y: Wiley.

Salameh, M. K., \& Jaber, M. Y. (2000). Economic production quantity model for items with imperfect quality. International Journal of Production Economics, 64(3), 59-64. http://dx.doi.org/10.1016/S0925-5273(99)00044-4

Shih, W. (1980). Optimal inventory policies when stockouts result from defective products. International Journal of Production Research, 18(6), 677-686. http://dx.doi.org/10.1080/00207548008919699

Silver, E. A. (1976). Establishing the reorder quantity when the amount received is uncertain. INFOR, 14(1), 32-39.

Wee, Hui-Ming., Wang, Wan-Tsu., \& Yang, Po-Chung. (2013). International Journal of Production Research. 51(6), 1869-1884. http://dx.doi.org/10.1080/00207543.2012.718453

Wolf, R. W. (1989). Stochastic Modeling and the theory of Queues. Prentice-Hall, Englewood Cliffs, NJ.

Yano, C. A., \& Lee, H. L. (1995). Lot sizing with random yields: A review. Operations Research, 43, 311-334. http://dx.doi.org/10.1287/opre.43.2.311

\section{Copyrights}

Copyright for this article is retained by the author(s), with first publication rights granted to the journal.

This is an open-access article distributed under the terms and conditions of the Creative Commons Attribution license (http://creativecommons.org/licenses/by/3.0/). 